\title{
Sentiment classification based on linguistic patterns in citation context
}

\author{
Mingyang Wang*, Dongtian Leng, Jinjin Ren, Yiming Zeng and \\ Guangsheng Chen \\ College of Information and Computer Engineering, Northeast Forestry University, Harbin 150040, People's Republic of China
}

\begin{abstract}
When citations occur, authors tend to express their emotions implicitly, which makes it difficult to identify the sentiment of citation context. However, authors will still use specific linguistic patterns to express their emotions in citation context. This article explores the linguistic patterns of emotional expression in citation context, and on this basis recognizes the sentimental polarity of citation context. Conditional random fields (CRF) model is introduced to annotate the logical relationship between syntactic structure and vocabularies in linguistic patterns. By analysing the effect of the generated CRF templates in classifying the subjective/objective sentences and the positive/negative emotional polarity in citation context, the role of linguistic patterns in classifying the citation sentiment is discussed. Experimental results show that the CRF model based on linguistic patterns is superior to the commonly used support vector machine (SVM) model in both subjective/objective and emotional polarity classification tasks. In the SVM model, the contextual information of citation context is considered by introducing one deep learning model of Word2vec. It shows that extracting linguistic patterns from the citation context helps reflect the way in which an author organizes his/her language in expressing his/her emotions. Extracting these linguistic patterns helps improve the performance of sentiment classification of citation context.
\end{abstract}

Keywords: Citation context, conditional random fields, linguistic patterns, sentiment classification, support vector machine.

TRADITIONAL citation analysis mainly focuses on the citation frequency of the cited documents, but ignores the content of citation context. This makes it difficult for traditional citation analysis to effectively acquire the citation sentiment to the cited documents. Thus it is not easy to effectively evaluate the value of the cited documents. The development of natural language processing technology has greatly encouraged research on citation context analysis $^{1-6}$. The recognition of citation sentiment embodied in citation context has become an important research topic in citation context analysis. Sentiment recognition

\footnotetext{
*For correspondence. (e-mail: wmynefu@163.com)
}

of citation context can help to reveal the motivation of researchers' citation and distinguish the value of the cited documents $^{7-11}$. However, sentiment recognition of citation context is much more complex than sentiment analysis of traditional text (such as micro-blog text). The citation context has its own characteristics. For example, when citations occur, an author usually implicitly expresses his/her emotions, especially his/her negative emotions. This greatly increases the difficulty of sentiment recognition of the cited context. How to recognize more accurately the implicit emotional tendency in citation context has become difficult in sentiment analysis of citation context.

At present, research on sentiment polarity recognition in citation context mainly focuses on the application of machine learning methods and selection of input features. Sentence subject ${ }^{12}$, clue words ${ }^{13-15}$, n-gram ${ }^{16,17}$, negative phrase $^{12,18}$ and dependency relations ${ }^{18}$ were extracted as inputs of different machine learning models to explore the role of these features in sentiment recognition of the cited context. Referring to sentiment lexical in other fields, researchers established the sentiment lexical of citation context in order to realize the sentiment recognition of citation context ${ }^{19}$. Although these features extract the syntactic structure from different aspects, they are relatively isolated and fail to fully examine the context information where these features exist and the semantic information brought about by a combination of features. Some researchers confirmed that adding contextual information in the citation sentence can help identify citation sentiment more accurately, especially to improve the recognition effect of negative sentiments ${ }^{18,20}$.

Recently, researchers found that authors of articles often used specific linguistic patterns to express their feelings to the citation context ${ }^{19,21,22}$. The linguistic patterns in citation context reflected the way in which an author organizes his/her language in expressing his/her emotions and contributed to fully examining the context information and semantic information. However, these linguistic patterns only contain relatively simple logical relationships between features or word patterns by n-gram. For example, only the logical relationship between sentiment words and negative words was considered. If there are negative words around sentiment words, polarity will be reversed $^{19}$. Through careful observation of the citation 
context, we found that there are more complex linguistic patterns in the citation context. Although no emotional words may appear in these linguistic patterns, they still show specific emotional tendencies through more complex feature combinations. In this article, we explore the complex linguistic patterns used to express implicit emotions in citation context, which include word patterns and structure patterns together, and extract the feature combination patterns in these linguistic patterns. It is hoped that through the effective expression of these complex feature combination patterns, the effect of sentiment recognition of citation context will be improved.

Conditional random field (CRF) is a discriminant probability model which has been widely used in natural language processing to annotate and partition sequential data $^{23}$. Compared with other discriminant models, the advantage of the CRF model is that it can fuse the context information of tagged data, effectively identify the logical structure between features, and take into account the complex dependencies between words ${ }^{24}$. Compared with hidden Markov model and other probabilistic graph models, the tag template provided by CRF has a greater ability to fuse related features, and can give full play to the advantages of multi-feature combination ${ }^{25}$. Therefore, the CRF model was introduced in this study to annotate the linguistic patterns in citation context, and to vectorize the logical structure between complex features in the linguistic patterns. By transforming these linguistic patterns into templates in CRF and using these templates to identify citation sentiment, we discuss the role of these complex linguistic patterns in sentiment recognition of citation context.

\section{Data source and pre-processing}

\section{Data source}

The original data used in this study are from the public data provided by Abu-Jbara et al. ${ }^{12}$ (http://clair.si.umich. edu/corpora/citation_sentiment umich.tar.gz). They had selected 30 articles from the ACL Anthology Network (AAN, including 19,000 Natural Language Processing (NLP) articles). These 30 articles were cited 3500 times by 1493 papers in the corpus. The citation text fragments corresponding to the 3500 citations were extracted, each of which contained four sentences: a sentence with obvious citation marks, the first two sentences before this sentence and the following one after this sentence in the citing papers. Finally, a corpus containing 3500 citation text fragments and 14,000 sentences was generated. Each sentence was marked as ' 1 ' or ' 0 ', according to whether it was helpful to sentiment judgment of the citation text fragment - ' 1 ' indicates that the sentence is helpful in identifying the citation sentiment, ' 0 ' indicates that it is not. The whole citation text fragment had two tags: (1) target tags: 1 - criticism, 2 - comparison, 3-use, $4-$ experience, 5 - basis, 6 - neutrality; (2) emotional polarity tags: 1 - neutrality, 2 - position, 3 - negative.

In the annotation of the corpus by Abu-Jbara et al. ${ }^{12}$, if the cited article is used by a third-party author, the cited document is marked as a positive citation. In fact, it is found that in this case the cited documents are mainly mentioned by third-party authors, which actually reflects a neutral citation. Based on this observation, we remarked the citation text fragment in the corpus. (a) If the citation text segment expresses a positive sentiment tendency towards the cited document or indicates that the cited document has been directly used, then it is marked as a positive citation. (b) If the citated document is compared with another article (possibly the same author's article) and to some extent considered better, the citation text segment will be marked as a positive citation. (c) If the limitation of the cited document is clearly pointed out in the citation text segment, the citation text segment is marked as a negative citation. (d) If the cited document is compared with another article (possibly the same author's article) and is considered poor to some extent, the citation text segment is marked as a negative citation. (e) If the citation text fragment only describes that the cited document has been used by third-party authors, the citation text segment is marked as neutral.

\section{Data pre-processing}

In the original citation text fragments, each fragment contains four sentences. Some of these sentences may not be helpful for sentiment classification of the citation text fragments. In order to improve the efficiency of subsequent sentiment classification, the sentences in the citation fragments were first pre-processed by eliminating the useless sentences. A new citation fragment corpus based on the remaining sentences was generated finally.

\section{A. Pre-processing of raw datasets}

(1) If the tags of four sentences in a citation text fragment are all labelled as ' 0 ', delete this citation text fragment because all the sentences in this text fragment cannot help judge their citation sentiment.

(2) Choose sentences marked ' 1 ' from each remaining citation text fragment, and form new citation text fragments on this basis. There are three types of new citation text fragments: (i) when the sentences labelled as ' 1 ', appear continuously in one citation text fragment, then all these sentences form a new text fragment together; (ii) when the sentence marked ' 1 ' does not appear continuously in one citation text fragment, it is merged with the sentence marked ' 0 ' in the middle into a new citation text fragment; (iii) when there is only one sentence marked as ' 1 ' in the citation text fragment, it is treated as a new citation text fragment. 


\section{RESEARCH ARTICLES}

(3) Since sentences in the citation text fragments are acquired through regular expressions, it is inevitable to obtain some sentences which do not conform to the syntactic structure (such as lack of verbs in the sentences). These sentences were deleted from the new citation fragment corpus. Finally, we obtained a corpus of 1260 citation text fragments with emotional polarity tags.

\section{B. Pre-processing of reference marking}

When writing academic papers, authors usually use special formats (such as parentheses) to label references. These tags are not part of the sentence and need to be processed. The processing steps to the reference tags in the corpus are as follows:

Step 1: Simplify the tags of references as: $<\mathrm{TREF}>$... $<\mathrm{TREF}>\rightarrow<\mathrm{TREF}>$; $\quad<\mathrm{REF}>\ldots<\mathrm{REF}>\rightarrow<\mathrm{REF}>$ (where TREF denotes the target reference; REF denotes the other references).

Step 2: For the continuous occurrence of multiple reference tags, they are further processed in two ways: (i) if $<$ TREF $>$ exists in the continuous occurrence of reference tags, those tags that appear continuously will be replaced by $<$ GTREF $>$; (ii) if $<$ TREF $>$ does not exist in the continuous occurrence of reference tags, these tags will be replaced by $<\mathrm{GREF}>$.

Step 3: Some reference tags do not act as a sentence component in citation text fragments, which should be deleted. As shown in Figure 1, the reference tag $<$ GTREF $>$ is not used as a sentence component and needs to be deleted. However, in order to not lose the location information of $<\mathrm{GTREF}>$, we need to track its location by syntactic parsing tree. The location of the target reference is marked by finding the first node of the noun phrase before $<$ GTREF $>$. For example, in Figure 1, after the nearest noun 'models' to the deleted $<\mathrm{GTREF}>$, a '*, symbol is added to mark the location of the target reference. In this way, the location information of $<$ GTREF $>$ will not be lost, and will help find the aspect words which

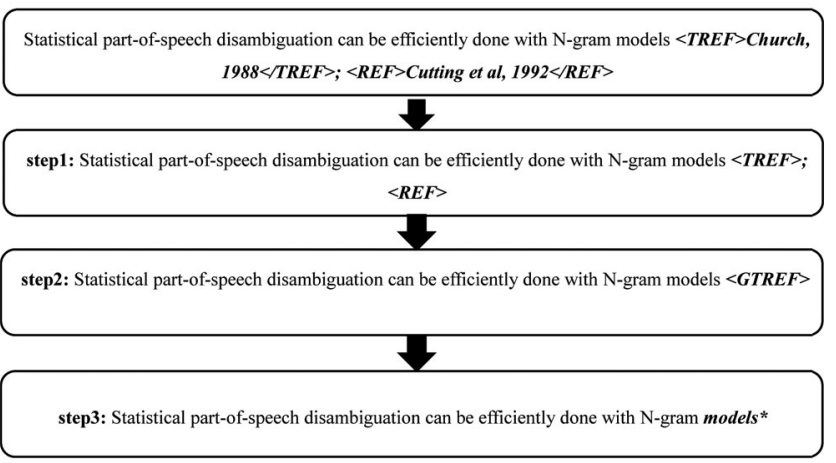

Figure 1. An example of pre-processing of reference marking. will be used in the following feature selection and annotation process.

After pre-processing of the citation text fragments, the sentence structure in the fragments is more standardized. Then we continue to extract the features suitable for citation sentiment classification tasks from citation text fragments.

\section{Feature selection and annotation}

On the basis of examining the semantic and syntactic structure of citation context, 10 features were extracted from the citation text fragments. A combination of these features was used to represent the linguistic patterns extracted from citation context. Table 1 gives the description of 10 selected features.

In Table 1, negative scope is considered to be one of the characteristics of citation emotional classification. The existence of negative words in sentences may reverse the emotional polarity of the text within the scope of negation. The part of a sentence that may change its emotional polarity under the influence of negative words is called the negative scope of the sentence ${ }^{26}$. In the task of citation sentiment classification, taking negative scope as one of the features helps identify the parts affected by negative words, which will help to effectively determine the emotional polarity of citation text fragments ${ }^{12,18,27}$. This article proposes seven rules for defining negative scope.

(1) If the main verb in a sentence is negated, the whole sentence is within the scope of negation.

(2) If the negative word is located in the clause of the sentence, its influence is limited to the clause (the coordinating conjunction is used as the boundary).

(3) If the negative word is located in the main sentence, the whole sentence is within the scope of negation (including clauses).

(4) If the negative word lies in a clause, the whole clause is within the scope of negation, excluding the subject clause (the subordinating conjunction is used as the boundary).

(5) If the subject of a sentence is in the negative range, the whole sentence is in the negative range.

(6) If the object of the main verb in a sentence is negated, the whole sentence is within the scope of negation; if it is not the main verb, the object is within the scope of negation (if the object is nothing, the scope of negation is the subject and verb of the sentence).

(7) If an adjective in a noun phrase is negated and acts as a predicative adjective, the scope of negation is the whole sentence; if it does not act as a predicative adjective, the scope of negation is the whole noun phrase. 
Table 1. Description of the 10 selected features

\begin{tabular}{|c|c|}
\hline Features & Description \\
\hline Word & Words in citation text fragments. \\
\hline Part-of-speech & Using Python Natural Language Toolkit (NLTK) to annotate the part-of-speech. \\
\hline Aspect word & $\begin{array}{l}\text { If }<\mathrm{TREF}>\text { exists in a sentence, find the noun phrase where }<\mathrm{TREF}>\text { is located and mark all the nouns in the } \\
\text { noun phrase as aspect words; if }<\mathrm{TREF}>\text { does not appear in the sentence but a symbol }(*) \text { does, find the } \\
\text { noun phrase where the symbol }(*) \text { is located and mark all the nouns in the noun phrase as aspect words. }\end{array}$ \\
\hline Evaluation word and its polarity & $\begin{array}{l}\text { (a) Words that directly reflect sentiment tendencies such as adjectives and adverbs, are marked as evaluation } \\
\text { words and labelled as positive or negative accordingly. Some verbs also have obvious sentiment tendency } \\
\text { by modifying aspect words. These are also detected and marked as evaluation words with positive or nega- } \\
\text { tive polarity accordingly. (b) Some words indirectly reflect sentiment tendencies in the citation text frag- } \\
\text { ments, such as some verbs. When the citing document (usually expressed in the first person, such as we, } \\
\text { our, this paper, etc.) is taken as the subject or object and the cited document correspondingly is taken as the } \\
\text { object or subject, the conjunctive verbs (such as use, application, etc.) between the subject and the object } \\
\text { are extracted as evaluation words, and the polarity is marked as neutral. }\end{array}$ \\
\hline Adversary word & Transitional conjunctions in sentences. \\
\hline Negative word & Negative words in sentences. \\
\hline Scope of negation & Part of a sentence that is affected by negative words and leads to a change in emotional polarity. \\
\hline Personal subject & Personal subject of the current document. \\
\hline Syntactic structure information & $\begin{array}{l}\text { Stanford Parser is used to obtain the following four kinds of syntactic dependencies in each sentence: subject- } \\
\text { predicate-object relationship of the sentence; active-passive relationship of the predicate verb; adverb- } \\
\text { adjective relationship; adjective-noun relationship. }\end{array}$ \\
\hline n-gram & $\begin{array}{l}\text { In this work } 3 \text {-gram was used because it could reflect the word patterns and capture longer technical terms, } \\
\text { which have been demonstrated to have a better effect }{ }^{18} \text {. }\end{array}$ \\
\hline
\end{tabular}

A combination of these features was input into CRF to express the linguistic patterns extracted from the citation fragments. Table 2 shows the annotations and descriptions of these features in CRF.

According to the features described in Table 1, 297 aspect words, 339 evaluation words (including 176 positive words, 67 negative words, 96 neutral words), 13 adversative words and 14 negative words were obtained by tagging of the citation text fragments. Table 3 gives examples of these words.

\section{Feature template construction}

When citing other works, an author usually expresses his/her citation sentiment implicitly, especially when negative citation occurs. However, analysis of the ways in which implicit sentiments are expressed in citation texts shows that the author tends to implicitly express his/her citation emotion through specific linguistic patterns. This makes the citation sentiment classification to be closely related to the effective recognition of linguistic patterns in citation text fragments. Through analysis of citation fragments corpus, we extracted several distinct linguistic patterns that the author uses to express implicit sentiment. These linguistic patterns reflect some complex logical combinations of the features extracted in this study. In order to test the role of linguistic patterns in classifying the citation sentiment, we extracted the logical combination of the features in linguistic patterns and on this basis generated the feature templates of CRF.

In this study, a two-stage CRF model was used to identify citation sentiment in citation context. In the first stage, the citation fragments were divided into subjective or objective levels to identify the sentiment sentences in them. The second layer further classified the subjective citation fragments to recognize the implicit sentiment polarity in the citation fragments. By observing the subjective/objective sentences and positive/negative sentiment sentences, the linguistic patterns were first extracted to generate CRF templates, which were used as inputs for the two-stage sentiment classification task.

\section{Templates for subjective/objective classification}

Three hundred citation text fragments with subjective and objective tags (ratio $1: 1$ ) were randomly selected. The subjectivity of citation text fragments was expressed by the following four linguistic patterns:

(1) FP + ISW + ISA (subject is the current citing literature + neutral evaluation word + aspect word).

(2) ISP + ISA (positive evaluation word + aspect word).

(3) NOP + ISA (negative evaluation word + aspect word).

(4) ISW + ISE + ISA (neutral evaluation word + the negation scope + aspect word).

Based on the above four linguistic patterns and other features that can be used for subjective/objective classification, we designed eight feature templates which have progressive and comparative relationships. When designing feature templates, if a feature is added to reduce the classification accuracy of the current template, it will not appear in the next feature template; if the two feature 
RESEARCH ARTICLES

Table 2. Annotation and description of 10 features in conditional random fields (CRF)

\begin{tabular}{lll}
\hline Annotation & \multicolumn{1}{c}{ Value } & \\
\hline Word (W) & All possible words & Current word \\
Part-of-speech (POS) & All possible parts-of-speech & Part-of-speech of current word \\
Aspect word (ASP) & ISA & Yes \\
& NOA & No \\
Evaluation word (SEN) & ISS (ISW; ISP; NOP) & Yes (neutral; positive; negative) \\
& NOS & No \\
Adversary word (TURN) & IST & Yes \\
& NOT & No \\
Negative word (NEG) & ISN & Yes \\
& NON & No \\
Scope of negation (NEGS) & ISE & Yes \\
& NOE & No \\
Personal subject (PS) & FP & Subject is the current citing document \\
& TP & Subject is not the current citing document \\
Syntactic structure information (SSI) & NP_N; NP_J; COM_N; COM_J; Noun object; adjective modifying noun object; nouns subject; adjec- & No \\
& VB_I; VB_P; VB_V & tive modifying noun subject; active verb; passive verb; adverb \\
\hline
\end{tabular}

ISA, Is aspect word; NOA, Not aspect word; ISS, Is evaluation word and its polarity; NOS, Not evaluation word; IST, Is adversary word; NOT, Not adversary word; ISN, Is negative word; NON, Not negative word; ISE, Is in the scope of negative; NOE, Not in the scope of negative.

Table 3. Words detected from the citation text fragments (in descending order of frequency)

\begin{tabular}{|c|c|c|}
\hline \multicolumn{2}{|l|}{ Features } & Words \\
\hline Aspect word & & $\begin{array}{l}\text { TREF, method, GTREF, approach, model, tagger, work, measure, technique, algorithm, parser, tagging, thesaurus, } \\
\text { similarity, system... }\end{array}$ \\
\hline \multirow[t]{3}{*}{$\begin{array}{l}\text { Evaluation } \\
\text { word }\end{array}$} & Positive & $\begin{array}{l}\text { Success, necessary, widely, extend, efficient, effective, high, useful, accuracy, improve, attention, motivate, crucial, } \\
\text { reasonable, flexibility... }\end{array}$ \\
\hline & Negative & $\begin{array}{l}\text { Problem, lack, low, complex, limitation, rare, restrict, unfortunately, weakness, suffer, fail, overlap, overtaken, by- } \\
\text { pass, unhelpful... }\end{array}$ \\
\hline & Neutral & Use, base, propose, apply, describe, include, show, compare, identify, develop, give, similar, define, extract, report... \\
\hline \multicolumn{2}{|c|}{ Adversative word } & However, even, although, but, even if, yet, whereas, though, even though, while, in spite of, instead, nevertheless \\
\hline \multicolumn{2}{|c|}{ Negative word } & Hardly, not, never, no, only, without, less, neither...nor..., no, longer, too...to..., none, few, nothing, rare \\
\hline
\end{tabular}

Table 4. Feature templates used for subjective/objective classification

\begin{tabular}{|c|c|c|}
\hline Template id & Feature template & Description \\
\hline$S_{0}$ & Word + part-of-speech & $\begin{array}{l}\text { This is a baseline template. It contains only two original features, } \\
\text { viz. word and part-of-speech. }\end{array}$ \\
\hline$S_{1}$ & Word + part-of-speech; evaluation word & On the basis of $S_{0}$, the features of evaluation words are added. \\
\hline$S_{2}$ & Word + part-of-speech; aspect word + evaluation word & $\begin{array}{l}\text { On the basis of } S_{1} \text {, the features of aspect words are added. This tem- } \\
\text { plate is used to test the co-occurrence relationship between aspect } \\
\text { words and evaluation words. }\end{array}$ \\
\hline$S_{3}$ & $\begin{array}{l}\text { Word + part-of-speech; aspect word + polarity of evalua- } \\
\text { tion word }\end{array}$ & $\begin{array}{l}\text { Compared with } S_{2} \text {, the characteristics of evaluation words are re- } \\
\text { placed by the polarity of evaluation words. The template reflects } \\
\text { the co-occurrence of aspect words and the polarity of evaluation } \\
\text { words, and ISP/NOP + ISA pattern. }\end{array}$ \\
\hline$S_{4}$ & $\begin{array}{l}\text { Word + part-of-speech; aspect word + polarity of evalua- } \\
\text { tion word; the person of subject }\end{array}$ & $\begin{array}{l}\text { On the basis of } S_{3} \text {, subject personal characteristics are added to re- } \\
\text { flect the FP + ISW + ISA pattern. }\end{array}$ \\
\hline$S_{5}$ & $\begin{array}{l}\text { Word + part-of-speech; aspect word + polarity of evalua- } \\
\text { tion word + scope of negation; person of subject }\end{array}$ & $\begin{array}{l}\text { On the basis of } S_{4} \text {, the negative range feature is added to reflect the } \\
\text { ISW + ISE + ISA pattern. }\end{array}$ \\
\hline$S_{6}$ & $\begin{array}{l}\text { Word + part-of-speech; aspect word + polarity of evalua- } \\
\text { tion word + scope of negation; the person of subject; } \\
\text { syntactic structure information }\end{array}$ & $\begin{array}{l}\text { On the basis of } S_{5} \text {, the features of syntactic structure are added to } \\
\text { reflect the subject-predicate-object relationship between sen- } \\
\text { tences and words, the active-passive relationship between predi- } \\
\text { cate verbs, and the modifier relationship between adjectives and } \\
\text { adverbs. }\end{array}$ \\
\hline$S_{7}$ & $\begin{array}{l}\text { Word + part-of-speech; aspect word + polarity of evalua- } \\
\text { tion word + scope of negation; person of subject; } \\
\text { 3-gram }\end{array}$ & $\begin{array}{l}\text { On the basis of } S_{5} \text {, the } 3 \text {-gram features are added to reflect the influ- } \\
\text { ence of relation between the front and back of words on the classi- } \\
\text { fication effect. }\end{array}$ \\
\hline
\end{tabular}


RESEARCH ARTICLES

Table 5. Feature templates used for polarity classification

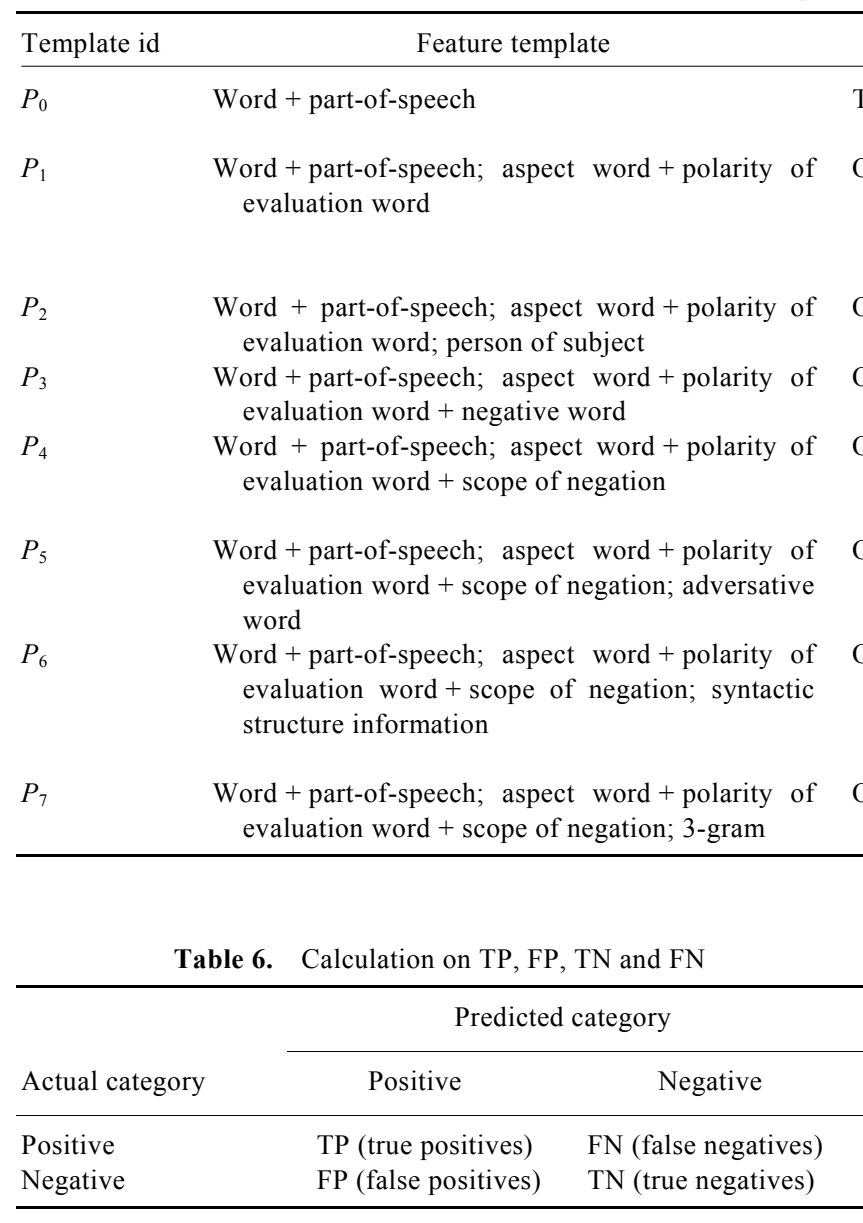

templates are comparative, the template with poor classification effect will not appear in the next feature template. Table 4 shows eight feature templates that were finally generated.

\section{Templates for sentiment polarity classification}

One hundred and fifty subjective sentences with sentiment polarity category labels (ratio between positive and negative is $2: 1$ ) were randomly selected. It was found that most of the positive or negative sentiments were expressed by the following seven linguistic patterns in citation text fragments.

For sentences with positive sentiment:

(1) FP + ISW + ISA (subject is the current citing literature + neutral evaluation word + aspect word).

(2) ISP + ISA (positive evaluation word + aspect word).

(3) ISP + ISW + ISA (positive evaluation word + neutral evaluation word + aspect word).

For sentences with negative sentiment:

(4) NOP + ISA (negative evaluation word + aspect word).

(5) ISW + ISE + ISA (neutral evaluation word + negation scope + aspect word).

(6) ISP + ISE + ISA (positive evaluation word + negation scope + aspect word).

(7) NOP + ISW + ISA (negative evaluation word + neutral evaluation word + aspect word).

Based on the above seven linguistic patterns and other features that can be used for polarity classification, eight feature templates with progressive and contrastive relationships were designed. Table 5 describes the eight feature templates for polarity classification.

\section{Experimental results and discussion}

Compared to the actual and predicted category results of the experimental samples, the experimental samples could be divided into four groups, viz. true positive (TP), false positive (FP), true negative (TN) and false negative (FN). Table 6 shows the calculation on TP, FP, TN and FN.

Based on TP, FP, TN and FN, we calculated three indices of precision $(P)$, recall $(R)$ and $F_{1}$-score $\left(F_{1}\right)$ to detect the classification performance.

$$
\begin{aligned}
& P=\mathrm{TP} /(\mathrm{TP}+\mathrm{FP}), \\
& R=\mathrm{TP} /(\mathrm{TP}+\mathrm{FN}), \\
& F_{1}=2 P R /(P+R) .
\end{aligned}
$$

Fivefold cross-validation was used to determine the final value of $P, R$ and $F_{1}$. 
Table 7. Subjective and objective classification results under CRF templates (\%)

\begin{tabular}{lccc}
\hline & \multicolumn{3}{c}{ Classification results } \\
\cline { 2 - 4 } Feature templates & Precision & Recall & $F_{1}$-score \\
\hline$S_{0}$ & $69.33(69.74)[69.53]$ & $70.66(68.36)[69.51]$ & $69.97(69.03)[69.50]$ \\
$S_{1}$ & $76.50(75.20)[75.85]$ & $74.69(76.76)[75.73]$ & $75.47(75.87)[75.67]$ \\
$S_{2}$ & $78.24(77.05)[77.65]$ & $76.34(78.39)[77.37]$ & $77.09(77.53)[77.31]$ \\
$S_{3}$ & $84.38(80.75)[82.56]$ & $79.72(85.04)[82.38]$ & $81.90(82.77)[82.34]$ \\
$S_{4}$ & $88.22(85.80)[87.01]$ & $85.47(88.33)[86.90]$ & $86.78(87.00)[86.89]$ \\
$S_{5}$ & $88.38(86.50)[87.44]$ & $86.21(\mathbf{8 8 . 4 6})[87.34]$ & $87.23(87.41)[87.32]$ \\
$S_{6}$ & $86.47(85.29)[85.88]$ & $85.27(86.40)[85.83]$ & $85.84(85.82)[85.83]$ \\
$S_{7}$ & $\mathbf{8 8 . 5 6}(\mathbf{8 8 . 4 2})[\mathbf{8 8 . 4 9}]$ & $\mathbf{8 8 . 4 0}(88.19)[\mathbf{8 8 . 3 0}]$ & $\mathbf{8 8 . 3 7}(\mathbf{8 8 . 1 8})[\mathbf{8 8 . 2 8}]$ \\
\hline
\end{tabular}

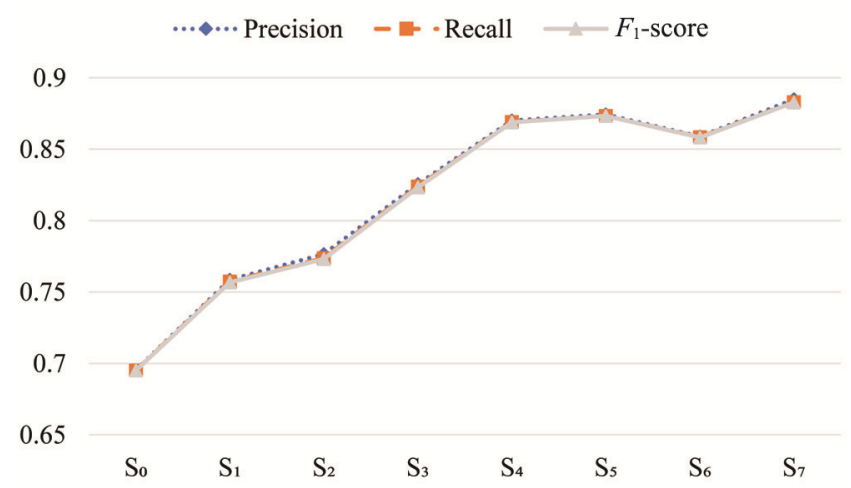

Figure 2. Subjective/objective classification results under CRF templates $(\%)$.

\section{Experimental results for subjective/objective classification}

Table 7 shows the final experimental results for subjective/objective classification. The three consecutive values in the cell correspond to: subjective classification result (objective classification result) [average value]. The average value is for subjective and objective classification results obtained under a template. The values shown in bold are the maximum in each column.

Figure 2 shows the curve of classification results in a more intuitive manner. Because the values of the three indices of $P, R$ and $F_{1}$ calculated under each feature template are very close, the three curves are basically the same; but this does not affect the comparison of classification results under each feature template. Since the index $F_{1}$ is the comprehensive result of $P$ and $R$, the value of $F_{1}$ was used to analyse the performance of subjective/objective classification under different CRF templates.

It can be seen that $F_{1}$ increases rapidly from template $S_{0}$ to $S_{5}$. It decreases slightly under $S_{6}$ and reaches a maximum under $S_{7}$. Compared with $S_{0}$, the increase of $F_{1}$ under template $S_{1}$ indicates that the evaluation words added in $S_{1}$ can assist in performing the subjective/objective classification. The value of $F_{1}$ increases from $S_{1}$ to $S_{3}$, which indicates that the co-occurrence of aspect words and evaluation words is helpful for classification, and it is more important to examine the polarity of evaluation words than whether these words appear. The value of $F_{1}$ keeps increasing from $S_{3}$ to $S_{5}$, indicating that the linguistic patterns extracted from the citation text fragments are of great significance to classify the subjective/objective citations. Also, the more perfect the linguistic pattern is, better will be the classification performance. The classification performance of $S_{6}$ decreases slightly, indicating that the information added by the syntactic structure may interfere with the existing features. $F_{1}$ reaches its maximum value under $S_{7}$, showing that adding 3-gram features to linguistic patterns is more conducive to subjective/ objective classification. Because CRF obtains the best performance under $S_{7}$, this has been chosen as the final template for subjective/objective classification.

\section{Experimental results for sentiment polarity classification}

Based on the constructed CRF feature templates for polarity classification, we identified the citation sentiment polarity of subjective sentences. Table 8 shows the experimental results for sentiment polarity classification. The three consecutive values in the cell correspond to: positive classification result (negative classification result) (average value). The average value is for positive/ negative classification results obtained under a template. The values shown in bold are the maximum in each column.

Table 8 shows that under each template, the classification performance of positive citation is significantly higher than that of negative citation, which makes it unreasonable to use the average value of positive and negative classification results to evaluate the classification performance. Here, the performance of positive and negative classification has been discussed under different templates to observe their influence on sentiment polarity classification. 
Table 8. Results of sentiment polarity classification under CRF templates (\%)

\begin{tabular}{lccc}
\hline & \multicolumn{3}{c}{ Classification results } \\
\cline { 2 - 4 } Feature templates & Precision & Recall & $F_{1}$-score \\
\hline$P_{0}$ & $82.65(67.66)[75.15]$ & $91.14(46.99)[69.06]$ & $86.55(54.13)[70.34]$ \\
$P_{1}$ & $88.06(81.37)[84.72]$ & $94.38(64.79)[79.59]$ & $91.07(71.67)[81.37]$ \\
$P_{2}$ & $88.22(80.61)[84.41]$ & $93.93(65.40)[79.66]$ & $90.93(71.62)[81.27]$ \\
$P_{3}$ & $89.37(86.70)[88.04]$ & $95.94(68.83)[82.39]$ & $92.52(76.52)[84.52]$ \\
$P_{4}$ & $\mathbf{9 2 . 1 3}(86.58)[89.35]$ & $95.53(77.66)[86.59]$ & $93.79(81.80)[87.80]$ \\
$P_{5}$ & $92.29(85.55)[88.92]$ & $95.08(\mathbf{7 8 . 2 5})[86.67]$ & $93.66(81.64)[87.65]$ \\
$P_{6}$ & $91.59(88.67)[\mathbf{9 0 . 1 3}]$ & $96.20(75.93)[86.06]$ & $\mathbf{9 3 . 8 2}(81.64)[87.73]$ \\
$P_{7}$ & $90.34(88.48)[89.41]$ & $\mathbf{9 6 . 4 0}(71.79)[84.10]$ & $93.25(79.05)[86.15]$ \\
\hline
\end{tabular}

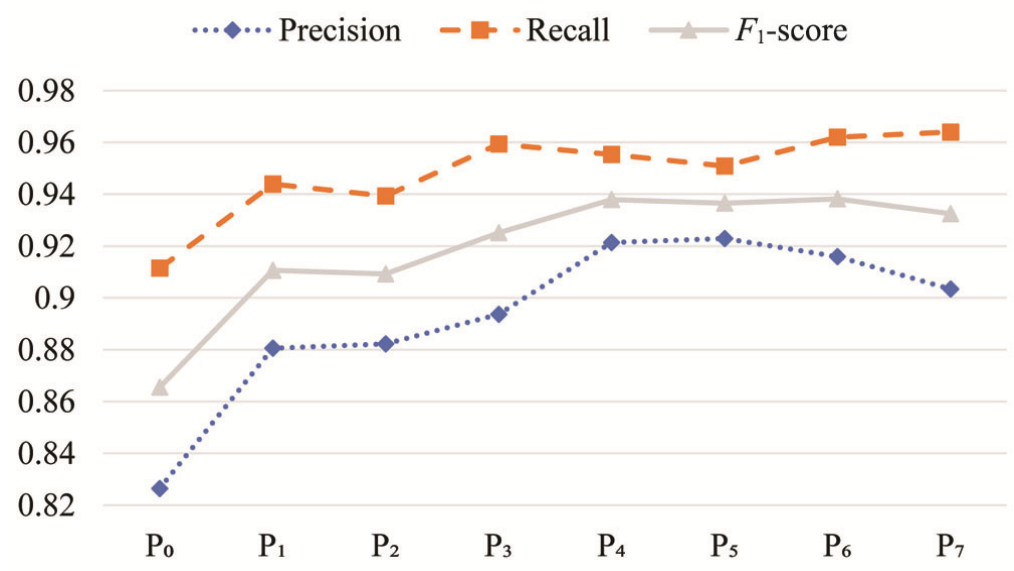

Figure 3. Positive classification results under CRF templates (\%).

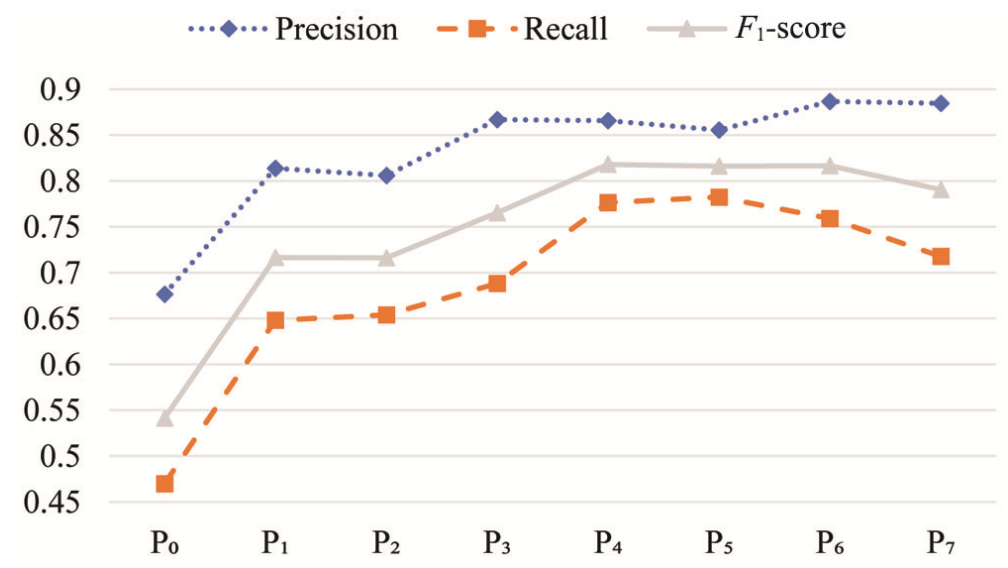

Figure 4. Negative classification results under CRF templates (\%).

Figures 3 and 4 show the results of positive and negative classification respectively. Also, the value of $F_{1}$ was used to analyse the performance of positive/negative classification under different CRF templates. The results show that in positive and negative citation classification, the change trend of $F_{1}$ values under each template is basically the same. $P_{1}-P_{4}$ are the feature templates constructed on linguistic patterns. The value of $F_{1}$ increases rapidly from $P_{1}$ to $P_{4}$, which indicates that feature combination based on linguistic patterns still plays an important role in citation polarity classification. Compared with $P_{1}$, the decrease in $F_{1}$ under $P_{2}$ shows that the person-subject feature added in $P_{2}$ has little effect on polarity classification. From $P_{3}$ to $P_{4}$, the value of $F_{1}$ increases significantly, indicating that negative cue information is important in citation polarity classification. In addition, relative to 
'whether there are negative words', 'whether they are within the scope of negation' is more important for negative cue information. The decrease in $F_{1}$ under $P_{5}$ to $P_{7}$ indicates that adding the features of adversative words, syntactic structure and 3-gram cannot further complement the templates generated by linguistic patterns. Since the performance of polarity classification under $P_{4}$ reaches its peak, this is chosen as the final template for CRF polarity classification.

\section{Classification results based on support vector machine model}

Support vector machine (SVM) model is one of the classification models which has been widely used in classifying the citation sentiment of citation context $\mathrm{t}^{28-31}$. In the present study, the SVM model was used to make a comparison with the proposed CRF-model to detect whether the CRF-based classification model could better identify the sentiment of citation text fragments. Word2vec, a deep learning model was introduced to distribute citation text fragments to generate structured vectors, which would be the input for SVM. Word2vec can realize the vectorization of words on the premise of considering the semantic, grammatical and contextual information of words ${ }^{32}$. The advantage of examining the context information of words makes Word2vec popular in topic detection $^{33}$, automatic summarization ${ }^{34,35}$, sentiment analysis ${ }^{36}$ and other application fields ${ }^{37}$. Therefore, by inputting the structured vectors generated by Word2vec, the classification model of SVM can contain as much semantic information as possible. The process of using Word2vec to generate the vectors of citation text fragment is as follows: the stop words were first deleted from the citation text fragments. The other words were trained into word vectors with different dimensions by Word2vec to detect the best dimensions of word distributed expression using Word2vec. Then, the vectorization of citation text fragment was obtained by linear superposition of word vectors located in the citation text fragment. The vectorized citation text fragments were input into the SVM model to complete the classification task. Two different kernels of linear and RBF were used in the SVM model to ensure a better classification effect.

Table 9 shows the results of subjectivity/objectivity and sentiment polarity classification of citation text fragments using the SVM model. $F_{1}$ value was used to evaluate the classification performance, which was calculated by five-fold cross-validation method. The classification results of positive and negative polarity have been discussed in classifying sentiment polarity. The two values in the cell in sentiment polarity classification correspond to: positive classification result (negative classification result). The values shown in bold are the maximum in each column.
The results show that in subjective/objective classification, performance of the SVM model based on RBF kernel function is better than that based on linear kernel function. While in sentiment polarity classification, classification effect of the SVM model based on linear kernel function is better than that based on RBF kernel function.

The best classification results based on the SVM model were extracted and compared with those based on the CRF model to explore whether the latter model has more advantages in classifying citation sentiment. The optimum experimental results of the two models correspond to the following experimental conditions:

(1) Subjective/objective classification: SVM (RBF kernel function, 50 dimensions); CRF (template $S_{7}$ ).

(2) Polarity classification: SVM (positive citation: RBF kernel function, 100 dimensions; negative citation: linear kernel function, 500 dimensions); CRF (template $P_{4}$ ).

Table 10 shows the optimal results of SVM and CRF models under subjectivity/objectivity and sentiment polarity classification. $F_{1}$ value was used to evaluate the classification performance, which was calculated by fivefold cross-validation method. The classification results of positive and negative citation have been discussed. The two values in the cell in sentiment polarity classification correspond to: positive classification result (negative classification result). The values shown in bold are the maximum in each column.

The CRF model is superior compared to the SVM model in both subjectivity/objectivity and sentiment polarity classification. In subjective/objective classification, $F_{1}$ value of CRF model is 10.2 percentage points higher than that of the SVM model; in classifying positive and negative citations, the $F_{1}$ value of CRF is 2.6 and 10.4 percentage points higher than that of SVM respectively. This shows that the study of linguistic patterns and negative scope is more helpful to reveal the implicit sentiment tendencies in citation context. The CRF model can

Table 9. Classification results of SVM model (\%)

\begin{tabular}{|c|c|c|c|c|}
\hline \multirow[b]{2}{*}{ Dimension } & \multicolumn{2}{|c|}{ Subjectivity/objectivity } & \multicolumn{2}{|c|}{$\begin{array}{l}\text { Sentiment polarity } \\
\text { (positive/negative) }\end{array}$} \\
\hline & Linear & $\mathrm{RBF}$ & Linear & $\mathrm{RBF}$ \\
\hline 50 & 65.6 & 78.1 & $87.8(57.6)$ & $89.6(58.4)$ \\
\hline 100 & 66.7 & 77.0 & $88.0(58.4)$ & $91.2(66.4)$ \\
\hline 200 & 71.5 & 77.3 & $89.2(69.6)$ & $90.2(62.0)$ \\
\hline 300 & 70.1 & 71.2 & $87.6(64.8)$ & $90.6(66.2)$ \\
\hline 400 & 71.8 & 73.5 & $88.4(64.6)$ & $91.0(67.4)$ \\
\hline 500 & 71.4 & 71.1 & $89.2(71.4)$ & $90.0(65.2)$ \\
\hline 600 & 71.4 & 71.8 & $87.2(69.4)$ & $88.8(54.4)$ \\
\hline 700 & 71.0 & 69.9 & $90.2(70.6)$ & $89.4(53.2)$ \\
\hline 800 & 72.7 & 68.5 & $87.8(65.6)$ & $90.2(54.0)$ \\
\hline 900 & 69.6 & 67.8 & $88.2(63.6)$ & $90.0(53.2)$ \\
\hline
\end{tabular}


RESEARCH ARTICLES

Table 10. Classification results of SVM and CRF models (\%)

\begin{tabular}{lccccccc}
\hline & \multicolumn{3}{c}{ Subjectivity/objectivity } & & \multicolumn{3}{c}{ Sentiment polarity (positive/negative) } \\
\cline { 2 - 4 } \cline { 6 - 8 } Model & $P$ & $R$ & $F_{1}$ & & $P$ & $R$ & $F_{1}$ \\
\hline SVM & 79.0 & 77.2 & 78.1 & & $85.0(67.6)$ & $98.4(75.8)$ & $91.2(71.4)$ \\
CRF & $\mathbf{8 8 . 5}$ & $\mathbf{8 8 . 3}$ & $\mathbf{8 8 . 3}$ & & $\mathbf{9 2 . 1 ( 8 6 . 6 )}$ & $\mathbf{9 5 . 5 ( 7 7 . 7 )}$ & $\mathbf{9 3 . 8 ( 8 1 . 8 )}$ \\
\hline
\end{tabular}

effectively express the more complex logical relationship between features in linguistic patterns. It also helps in sentiment recognition and classification of citation context.

\section{Conclusion}

Citation sentiment classification is one of the basic approaches of citation context analysis. It can excavate the citation motivation of authors and help generate more scientific abstracts. Authors of citing articles often express their citation sentiment implicitly in the citation context, which increases the difficulty of sentiment recognition and classification of citation context. Analysis of citation context showed that an author usually expresses his/her sentiments implicitly through certain linguistic patterns. These linguistic patterns contain more complex logical relationships among words. On this basis, we have used the CRF model in this study, which can effectively annotate the sequential patterns in natural language. The CRF model was used to annotate the linguistic patterns appearing in the citation context, and to reflect the complex logical relationship among words in these patterns. On these annotated CRF templates, the classification of subjectivity/objectivity and positive/negative polarity of citation context was realized, and the influence of linguistic patterns on citation sentiment recognition was discussed.

The experimental results show that the CRF model achieves better results in both subjective/objective classification and positive/negative polarity classification compared to the SVM model. It shows that the combination features based on linguistic patterns are more helpful in identifying the implicit sentiments in citation context. Although the input fragment vectors in the SVM model also fuse the context information of citation context as much as possible, the recognition performance of citation sentiment is lower than that of the CRF model. This indicates that the extraction of linguistic patterns in citation context and the use of CRF to achieve the expression of more complex logical structures in these patterns can better capture the language organization patterns used by the authors, thus helping to identify the implicit sentiments in citation context.

1. Garfield, E., Journal impact factor: a brief review. Canadian Medical Association or its licensors. Can. Med. Assoc. J., 1999, 161(8), 979-980.
2. Seglen, P. O., Why the impact factor of journals should not be used for evaluating research. BMJ, 1997, 314(7079), 497.

3. Fu, H. Z. and Ho, Y. S., Collaborative characteristics and networks of national, institutional and individual contributors using highly cited articles in environmental engineering in Science Citation Index Expanded. Curr. Sci., 2018, 115(3), 410-421.

4. Nicholson, J. M. and Ioannidis, J. P. A., Research grants: conform and be funded. Nature, 2012, 492(7427), 34-36.

5. Saggion, H. and Ronzano, F., Scholarly data mining: making sense of scientific literature. In ACM/IEEE Joint Conference on Digital Libraries, Toronto, Canada, 2017, pp. 346-347.

6. Jha, R. et al., NLP-driven citation analysis for scientometrics. Nat. Lang. Eng., 2017, 23(1), 93-130.

7. Garfield, E., Is citation analysis a legitimate evaluation tool? Scientometrics, 1979, 1(4), 359-375.

8. Hernández-Alvarez, M. and Gomez, J. M., Survey about citation context analysis: tasks, techniques, and resources. Nat. Lang. Eng., 2016, 22(3), 327-349.

9. Marder, E., Kettenmann, H. and Grillner, S., Impacting our young. Proc. Natl. Acad. Sci. USA, 2010, 107(50), 21233.

10. Ghosh, S., Das, D. and Chakraborty, T., Determining sentiment in citation text and analyzing its impact on the proposed ranking index. In International Conference on Intelligent Text Processing and Computational Linguistics, Springer, Cham, Switzerland, 2016, pp. 292-306.

11. Sendhilkumar, S., Elakkiya, E. and Mahalakshmi, G. S., Citation semantic based approaches to identify article quality. In Proceedings of International Conference ICCSEA, Delhi, India, 2013, pp. 411-420.

12. Abu-Jbara, A., Ezra, J. and Radev, D., Purpose and polarity of citation: towards NLP-based bibliometrics. In Proceedings of the 2013 Conference of the North American Chapter of the Association for Computational Linguistics: Human Language Technologies, Atlanta, 2013, pp. 596-606.

13. Teufel, S., Siddharthan, A. and Tidhar, D., Automatic classification of citation function. In Proceedings of the 2006 Conference on Empirical Methods in Natural Language Processing. Association for Computational Linguistics, Sydney, Australia, 2006, pp. $103-110$.

14. Goodarzi, M., Mahmoudi, M. T. and Zamani, R., A framework for sentiment analysis on schema-based research context via lexica analysis. In IEEE 7th International Symposium on Telecommunications, 2014, pp. 405-411.

15. Parthasarathy, G. and Tomar, D. C., Sentiment analyzer: analysis of journal citations from citation databases. In IEEE 5th International Conference - Confluence the Next Generation Information Technology Summit (Confluence), 2014, pp. 923-928.

16. Kim, I. C. and Thoma, G. R., Automated classification of author's sentiment in citation using machine learning techniques: a preliminary study. In IEEE Conference on Computational Intelligence in Bioinformatics and Computational Biology, 2015, pp. 1-7.

17. Sula, C. A. and Miller, M., Citations, context, and humanistic discourse: Toward automatic extraction and classification. Lit. Linguist. Comput., 2014, 29(3), 452-464.

18. Athar, A., Sentiment analysis of citations using sentence structurebased features. In Proceedings of the ACL 2011 Student Session. 
Association for Computational Linguistics, Portland, Oregon, 2011, pp. 81-87.

19. Xu, J. et al., Citation sentiment analysis in clinical trial papers. In AMIA Annual Symposium Proceedings. American Medical Informatics Association, San Francisco, California, 2015, pp. 1334-1341.

20. Athar, A. and Teufel, S., Context-enhanced citation sentiment detection. In Proceedings of the 2012 Conference of the North American Chapter of the Association for Computational Linguistics: Human Language Technologies. Association for Computational Linguistics, Montreal, Canada, 2012, pp. 597-601.

21. Bertin, M. et al., The linguistic patterns and rhetorical structure of citation context: an approach using n-grams. Scientometrics, 2016, 109(3), 1417-1434.

22. Ikram, M. T. and Afzal, M. T., Aspect based citation sentiment analysis using linguistic patterns for better comprehension of scientific knowledge. Scientometrics, 2019, 119(1), 73-95.

23. Lafferty, J., McCallum, A. and Pereira, F. C. N., Conditional random fields: probabilistic models for segmenting and labeling sequence data. In Proceedings of the 18th International Conference on Machine Learning, Williamstown, MA, USA, 2001, pp. 282-289.

24. Li, X. and Li, J., Sentiment classification and strength analysis method based on three-layered conditional random fields. Appl. Res. Comput., 2017, 34(4), 986-990.

25. Shi, L. et al., Application of CRF and SVM based semi-supervised learning for semantic labeling of environments. In IEEE 12th International Conference on Control Automation Robotics \& Vision, Guangzhou, China, 2012, pp. 835-840.

26. Horn, L. R., A natural history of negation. J. Linguist., 1989 , 56(3), 426-433.

27. Morante, R., Schrauwen, S. and Daelemans, W., Annotation of negation cues and their scope: Guidelines v1. Computational linguistics and psycholinguistics technical report series, University of Antwerp, Antwerp, CTRS-003, 2011.

28. Hernandez-Alvarez, M., Soriano, J. M. G. and Martínez-Barco, P., Citation function, polarity and influence classification. Nat. Lang. Eng., 2017, 23(4), 561-588.

29. Hernández-Alvarez, M. and Gómez, J. M., Citation impact categorization: for scientific literature. In IEEE 18th International Confe- rence on Computational Science and Engineering, Porto, Portugal, 2015, pp. 307-313.

30. Hernández Álvarez, M., Gómez, J. M. and Martínez-Barco, P., Annotated corpus for citation context analysis. Latin-Am. J. Comput., 2017, 3(1), 35-41.

31. Ma, Z., Nam, J. and Weihe, K., Improve sentiment analysis of citations with author modelling. In Proceedings of the 7th Workshop on Computational Approaches to Subjectivity, Sentiment and Social Media Analysis, San Diego, California, 2016, pp. 122-127.

32. Mikolov, T. et al., Distributed representations of words and phrases and their compositionality. Adv. Neural Inf. Process. Syst., 2013, 2, 3111-3119.

33. Yan, D., Hua, E. and Hu, B., An improved single-pass algorithm for Chinese microblog topic detection and tracking. In IEEE International Congress on Big Data, Washington DC, 2016, pp. 251258.

34. Li, C. et al., LDA meets Word2Vec: a novel model for academic abstract clustering. In Companion of the Web Conference 2018, International World Wide Web Conferences Steering Committee, Lyon, France, 2018, pp. 1699-1706.

35. Chengzhang, X. and Dan, L., Chinese text summarization algorithm based on Word2vec. J. Phys.: Conf. Ser., 2018, 976(1), 012006.

36. Fauzi, M. A., Word2Vec model for sentiment analysis of product reviews in Indonesian language. Int. J. Electr. Comput. Eng., 2019, 9(1), 525-530.

37. Yao, Y. et al., Sensing spatial distribution of urban land use by integrating points-of-interest and Google Word2Vec model. Int. J. Geogr. Inf. Sci., 2017, 31, 825-848.

ACKNOWLEDGEMENTS. This work was supported by the National Natural Science Foundation of China (Grant No. 71473034; 717D1063), and the financial assistance from Postdoctoral Scientific Research Developmental Fund of Heilongjiang Province, China (Grant No. LBH-Q16003).

Received 9 May 2019; revised accepted 30 May 2019

doi: $10.18520 / \mathrm{cs} / \mathrm{v} 117 / \mathrm{i4} / 606-616$ 